\section{S7 HIGH MORTALITY FROM INVASIVE PNEUMOCOCCAL PNEUMONIA IN THE ERA OF VACCINE PREVENTABLE DISEASE}

K Ferguson, M Wilczynska. Hairmyres Hospital, Renfrew, UK

\subsection{6/thoraxjnl-2017-210983.13}

Introduction and Objectives Bacteraemia secondary to pneumococcal pneumonia is the most common presentation of invasive pneumococcal disease (IPD) and is associated with high mortality rates. ${ }^{1}$ We conducted this study to evaluate differences in the process of care and outcome in patients with community-acquired invasive pneumococcal pneumonia (CAIPP) depending on age, co-morbidities and vaccination status.

Methods This was a retrospective study that analysed the data for patients with CAIPP who were hospitalised in 2016 at NHS Lanarkshire hospitals.

Results Forty-five of 60 patients with pneumococcal bacteraemia had IPD secondary to community-acquired pneumonia. The mean age of the patients was 61 years (17-101). The CURB 65 score was $0-1$ in 55\% patients, 2 in $12 \%$ and $3-5$ in $33 \%$. Overall 30 days mortality was $22 \%$. The odds ratio of death within 30 days from CAIPP was 15.9 (95\% CI: 1.8140, $\mathrm{p}=0.012$ ) among those who had any co-morbidity involving major organ (cardiac, respiratory, renal or liver failure). Thirty days mortality showed the strongest positive association with age $(r=0.46, p=0.001)$ therefore patients were divided into two groups according to their age (Table 1). There was no difference between groups in time to reach diagnosis or initiate treatment. Within 4 hours of admission, a chest $\mathrm{x}$-ray was obtained in $88 \%$ in Group 1 vs. $89 \%$ in Group $2(p=N S)$, and the first dose of antibiotics was administered within 4 hours in 90\% in Group 1 vs. 94\% in Group $2(\mathrm{p}=\mathrm{NS}) .79 \%$ patients in Group 2 had at least one major co-morbidity vs. $46 \%$ in Group $1(\mathrm{p}=0.012)$. Group 2 had significantly higher 30 days mortality than group $1,47 \%$ vs $3.8 \%(\mathrm{p}=0.002)$. In the Group 2, among deceased 44\% (4 of 9) have not received pneumococcal vaccination. Only 4 of 26 patients in Group 1 were vaccinated against Streptococcus pneumonia.

Conclusions Clinical significance of IPP is underestimated in older people. Despite satisfactory initial assessment and prompt treatment mortality remains very high. That could be associated with the presence of co-morbidities and insufficient level of pneumococcal vaccination.

\section{REFERENCE}

1. Ludwig $E$ et al. The remaining challenges of pneumococcal disease in adults. Eur Respir Rev 2012;21:57-65.

\section{S8 RISK STRATIFICATION IN COMMUNITY ACQUIRED PNEUMONIA - CURB65, SIRS OR QSOFA? A RETROSPECTIVE ANALYSIS}

${ }^{1}$ DPS Dosanjh, ${ }^{1} \mathrm{~F}$ Grudzinska, ${ }^{1} \mathrm{~K}$ Aldridge, ${ }^{2} \mathrm{~S}$ Hughes, ${ }^{1} \mathrm{D}$ Thickett. ${ }^{1}$ Institute of Inflammation and Ageing, University of Birmingham, Birmingham, UK; ${ }^{2}$ University Hospitals NHS Foundation Trust, Birmingham, UK

10.1136/thoraxjnl-2017-210983.14

Introduction and Objectives The British Thoracic Society and National Institute for Health and Care Excellence recommend
Abstract S7 Table 1 Demographics and clinical data for group 1 and group 2.

\begin{tabular}{|c|c|c|}
\hline \multirow[t]{2}{*}{ Variables } & $\begin{array}{l}\text { Group } 1 \\
\text { (<65 years old) }\end{array}$ & $\begin{array}{l}\text { Group } 2 \\
\text { ( } \geq 65 \text { years old })\end{array}$ \\
\hline & $n=26$ & $n=19$ \\
\hline Age years (mean) & 47.9 & 81 \\
\hline Females (n) & 9 & 12 \\
\hline CRP mg/dL (mean) & 254 & 214 \\
\hline WBC $\times 10^{\wedge} 9 / L($ mean $)$ & 17.9 & 13.8 \\
\hline Chest x-ray: & 62 & 63 \\
\hline -lobar consolidation (\%) & 26 & 31 \\
\hline -multilobar consolidation (\%) & 12 & 6 \\
\hline \multicolumn{3}{|l|}{-consolidation and pleural effusion (\%) } \\
\hline Hospitalisation (days)* & 8.6 & 9.6 \\
\hline 30 days mortality (n) & 1 & 9 \\
\hline 30 days readmission $(n)$ & 2 & 2 \\
\hline Door to antibiotics time $(\min )$ & 103.5 & 99 \\
\hline Door to chest x-ray time ( $\min )$ & 146 & 101 \\
\hline Antibiotics duration (days)* & 12.7 & 9.3 \\
\hline $\begin{array}{l}\text { Antibiotics in the community before } \\
\text { admission (n) }\end{array}$ & 3 & 1 \\
\hline $\begin{array}{l}\text { Antibiotics according to local guidelines } \\
(\%)\end{array}$ & 53 & 36 \\
\hline
\end{tabular}

the CURB65 for severity assessment in community acquired pneumonia (CAP). The Third International Consensus Definitions for Sepsis and Septic Shock however, state that the qSOFA (quick Sequential Organ Failure Assessment) should be used to identify those with infection who are likely to have poor outcomes. This superseded the SIRS (Systemic Inflammatory Response Syndrome) criteria. There is therefore no clear international consensus regarding how severity of CAP should be assessed outside the critical care setting. We retrospectively evaluated the ability of the CURB65, SIRS and qSOFA scores to predict 30 day mortality in patients with CAP.

Methods Adults admitted to the Queen Elizabeth Hospital Birmingham, with CAP, between 10/2014 and 01/2016 were included. Radiology, admission clerkings and electronic patient records were reviewed to confirm pneumonia and calculate the scores. Cases were excluded if there was no radiological confirmation, or if they had hospital-acquired pneumonia.

Results 1545 patients were included in the final analysis (mean age 72, 30 day mortality 19.0\%, 49.1\% female). All scoring systems enabled stratification according to increasing risk of 30 day mortality. CURB65: $0 \%-3.5 \%, 1 \%-11.5 \%$; $2 \%-18.5 \% ; \quad 3 \%-27.1 \% ; \quad 4 \%-42.7 \%$ and $\quad 5 \%-42.1 \%$ $(\mathrm{p}<0.001)$. SIRS: $0 \%-10.4 \% ; 1 \%-13.5 \% ; 2 \%-18.1 \% ; 3 \%-$ $22.5 \% ; \quad 4 \%-32.5 \% \quad(\mathrm{p}<0.001)$. qSOFA: $0 \%-11.9 \% ; \quad 1 \%-$ $17.9 \% ; 2 \%-30.1 \% ; 3 \%-47.2 \%(\mathrm{p}<0.001)$. Receiver operator characteristic curves calculated to determine the accuracy with which the scores were able to predict 30 day mortality, revealed areas under the curve of $0.69,0.60$ and 0.63 for CURB65, SIRS and qSOFA respectively. Using the established cut-offs of CURB65 $\geq 2$, SIRS $\geq 2$ and qSOFA $\geq 2$, sensitivities, specificities, negative and positive predictive values for prediction of 30 day mortality were calculated (Table 1). Of those that died within 30 days, qSOFA $\geq 2$ correctly identified 
$40.5 \%$ as high risk, compared to $79.5 \%$ and $84.9 \%$ using the SIRS criteria and CURB65 respectively.

Conclusions All three scoring systems can stratify according to risk of 30 day mortality, though none of them are particularly accurate. qSOFA has poor sensitivity, and may underestimate severity and risk of 30 day mortality in CAP. New assessment tools to accurately identify CAP patients at increased risk of poor outcomes are urgently required.

\begin{tabular}{|c|c|c|c|c|c|c|}
\hline Score & $\begin{array}{l}\text { Sensitivity } \\
(\%)\end{array}$ & $\begin{array}{l}\text { Specificity } \\
(\%)\end{array}$ & $\begin{array}{l}\text { NPV } \\
(\%)\end{array}$ & $\begin{array}{l}\text { PPV } \\
(\%)\end{array}$ & $\begin{array}{l}\text { Negative } \\
\text { LR }\end{array}$ & $\begin{array}{l}\text { Positive } \\
\text { LR }\end{array}$ \\
\hline CURB65 & 84.9 & 40 & 91.5 & 25.9 & 0.38 & 1.42 \\
\hline SIRS & 79.5 & 32.5 & 87.3 & 21.4 & 0.63 & 1.18 \\
\hline qSOFA & 40.5 & 79.5 & 84.3 & 32.9 & 0.75 & 1.98 \\
\hline
\end{tabular}

NPV - negative predictive value, PPV - positive predictive value, LR - Likelihood ratio

\section{S9 $\quad$ INVASION VERSUS OVERGROWTH: UNDERSTANDING WHY RESPIRATORY PATHOGENS COLONISE THE MOUTH PRIOR TO DEVELOPMENT OF PNEUMONIA}

${ }^{1} \mathrm{VC}$ Ewan, ${ }^{1} \mathrm{~W}$ Reid, ${ }^{1} \mathrm{M}$ Shirley, ${ }^{2} \mathrm{~W}$ Wade, ${ }^{1} \mathrm{SP}$ Rushton, ${ }^{1} \mathrm{AJ}$ Simpson. ${ }^{1}$ Newcastle University, Newcastle upon Tyne, UK; ${ }^{2}$ Queen Mary University of London, London, UK

\subsection{6/thoraxjnl-2017-210983.15}

Introduction The presence of respiratory pathogens on oral surfaces is a risk factor for pneumonia. Understanding why non-oral respiratory pathogens appear is crucial in planning interventions to manipulate the oral microbiota to prevent pneumonia. We sought to understand whether respiratory pathogens were associated with reduction in oral bacterial diversity (invasion hypothesis) or no change in diversity (overgrowth hypothesis).

Methods We analysed extracted DNA from 167 throat samples from 53 hospitalised older patients with hip fracture using next generation sequencing (Lib-L chemistry, mothur). Occurrence of respiratory tract infection (RTI, clinician-initiated antibiotic for chest infection) within 3 months of discharge was noted via case notes and telephone call to General Practitioner. We used linear mixed effect modelling in $\mathrm{R}$ (nlme package) to investigate the association between relative abundance of respiratory pathogens and species richness, with patient as the random effect. We used correspondence analysis (CA) to analyse beta-diversity (vegan package).

Results Respiratory pathogens (Haemophilus influenzae, Staphylococcus aureus, Enterobactericeae) were present in $38 / 167$ samples (23\%). Higher relative abundances of respiratory pathogens were not significantly associated with sample diversity $(\mathrm{t}=-1.400575, \mathrm{p}=0.1641)$. Moreover, mixed effect models demonstrated no increase in relative abundances of respiratory pathogens over time in individual patients whilst in hospital $(\mathrm{t}=-0.206605, \mathrm{p}=0.8367)$. While RTI was associated with higher relative abundances of respiratory pathogens $(t=1.9502718, p=0.0567)$, there was no association between RTI and oropharyngeal species richness $(t=-0.361164$, $\mathrm{p}=0.7195$ ).

Conclusion There was no association between detection of respiratory pathogens and oropharyngeal species diversity. These Results support the overgrowth, rather than the invasion, hypothesis, and larger studies to explore frequency of oral clearance in conjunction with the oral microbiota are warranted. In addition, the lack of change over time in relative abundances of respiratory pathogens suggests that the exposure to the hospital environment is not a major driver in the appearance of these organisms.

\section{S10 SUPPRESSION OF MACROPHAGE INFLAMMATORY RESPONSES TO STREPTOCOCCUS PNEUMONIAE BY REGULATORY T CELLS}

G Szylar, J Brown. University College London, London, UK

\subsection{6/thoraxjnl-2017-210983.16}

Background The highly inflammatory immune response to Streptococcus pneumoniae infection can result in complications such as sepsis and Acute Respiratory Distress Syndrome. Macrophages are an important source of the inflammatory cytokines that activate epithelial and endothelial cells, resulting in a loss of barrier integrity. Regulatory T cells (Tregs) are a population of anti-inflammatory cells that modulate macrophage activity and are protective against invasive pneumococcal disease in mice. ${ }^{1,2}$

Aims To characterise the in vitro effects of Tregs on the macrophage inflammatory response to $S$. pneumoniae and to observe Treg recruitment to the site of intradermal injection of UV-killed S. pneumoniae in a human model.

Results Preliminary data suggest that co-culture of human monocyte-derived macrophages (MDMs) with $\mathrm{CD} 4{ }^{+} \mathrm{CD} 25^{+} \mathrm{CD} 127^{-}$Tregs reduced MDM TNF $\alpha$ production by at least $45 \%$ (One-way ANOVA $\mathrm{p}<0.01$ ) and IL-6 production by at least 52\% (One-way ANOVA p<0.01) 72 hours after initial infection with $S$. pneumoniae TIGR4 strain (MOI of 2, ratio of 1 Treg to $3 \mathrm{MDMs}$ ). Separation of Tregs from the MDMs during co-culture using transwell inserts prevented the suppressive effects of the Tregs. Using a novel human model of S. pneumoniae challenge involving intradermal injection of UV-killed S. pneumoniae into the forearm of healthy volunteers, we demonstrated that Tregs accumulated at the site of injection within 48 hours, increasing from undetectable Treg population at 4 hours to constituting approximately $33 \%$ of CD4 cells by 48 hours.

Conclusion Preliminary data suggest that Tregs modulate the MDM inflammatory response to $S$. pneumoniae in a contactdependent manner, and track to the site of intradermal injection of the UV-killed bacteria in human volunteers.

\section{REFERENCES}

1. Tiemessen MM, Jagger $A L$, Evans HG, van Herwijnen MJ, John S, Taams LS. CD4 +CD25+FoxP3+ regulatory $T$ cells induce alternative activation of human monocytes/macrophages. Proc Natl Acad Sci 2007;104(49):19446-51.

2. Neill DR, Fernandes VE, Wisby L, Haynes AR, Ferreira DM, Laher A, Strickland N, Gordon SB, Denny P, Kadioglu A, Andrew PW. T regulatory cells control susceptibility to invasive pneumococcal pneumonia in mice. PLoS Pathog 2012;8(4): e1002660. 